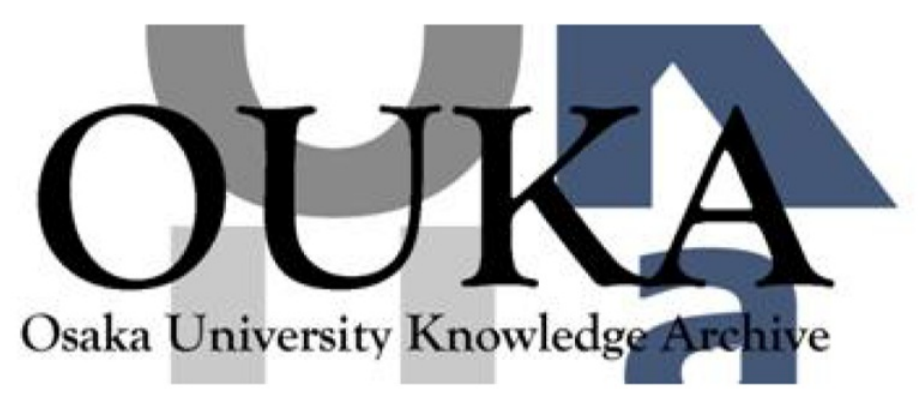

\begin{tabular}{|c|c|}
\hline Title & $\begin{array}{l}\text { Evidence of relativistic laser beam } \\
\text { filamentation in back-reflected images }\end{array}$ \\
\hline Author (s) & Tanaka, K. A. ; Allen, M. M. ; Pukhov, A. et al. \\
\hline Citation & Physical Review E. 62(2) p. 2672-p. 2677 \\
\hline Issue Date & $2000-08$ \\
\hline oaire:version & VoR \\
\hline URL & https://hdl. handle. net/11094/3242 \\
\hline rights & $\begin{array}{l}\text { Tanaka, K. A., Allen, M.M., Pukhov, A., Kodama, } \\
\text { R., Fujita, H. Kato, Y., Kawasaki, T., } \\
\text { Kitagawa, Y., Mima, K., Morio, N., Shi'raga, H. , } \\
\text { Iwata, M. 'Miyakoshi, T., Yamanaka, T. } \\
\text { Physical Review E, 62, 2, 2672-2677, 2000-08. } \\
\text { "Copyright } 2000 \text { by the American Physical } \\
\text { Society." }\end{array}$ \\
\hline Note & \\
\hline
\end{tabular}

Osaka University Knowledge Archive : OUKA

https://ir. Library. osaka-u. ac. jp/

Osaka University 


\title{
Evidence of relativistic laser beam filamentation in back-reflected images
}

\author{
K. A. Tanaka, ${ }^{*}$ M. M. Allen, ${ }^{\dagger}$ A. Pukhov, ${ }^{\ddagger}$ R. Kodama, H. Fujita, Y. Kato ${ }^{\S}$ T. Kawasaki, Y. Kitagawa, K. Mima, \\ N. Morio, H. Shiraga, M. Iwata, T. Miyakoshi, and T. Yamanaka \\ Institute of Laser Engineering, Osaka University, Yamada-oka 2-6, Suita, Osaka 565-0871, Japan
}

(Received 19 August 1999; revised manuscript received 2 March 2000)

\begin{abstract}
The back-reflected image of a $100 \mathrm{TW}$ laser incident on a long scale length plasma is measured. The plasma is deliberately preformed on a solid planar target in a controlled way. Multiple highly intense spots are observed inside the original focal spot. These spots could be the experimental evidence for the laser beam relativistic filamentation in the plasma. Three-dimensional particle-in-cell (PIC) simulations for parameters close to the experimental values are performed. The experimental observations and the filamentation dynamics obtained in the PIC simulations are in a good agreement.
\end{abstract}

PACS number(s): 52.40.Nk, 52.60.+h, 52.70.Nc, 52.75.Di

With the development of chirped pulse amplification (CPA) and multiterawatt laser systems [1,2], it is possible now to study the interaction of laser light with matter at ultrahigh intensities, $I>10^{18} \mathrm{~W} / \mathrm{cm}^{2}$. Electrons quiver in these laser pulses at relativistic energies. These studies are important for an understanding of basic physics and plasma phenomena in this new regime. One of the main driving applications for these laser pulses is the concept of fast ignition [3] in inertial confinement fusion (ICF). The fast ignition requires a channeling laser pulse to propagate as close as possible to the dense core of a fusion capsule, which has been compressed in the conventional manner. The characteristics of this channel formation are one of the key issues for fast ignitor research.

One option for the laser beam channeling through plasma is to use the relativistic self-focusing effect. The theory [4-6] predicts, and experiments with underdense gas jets [7-9] confirm, that the self-focusing occurs if the laser power exceeds the critical power given by

$$
P_{\mathrm{cr}}=17\left(\omega / \omega_{p}\right)^{2} \mathrm{GW},
$$

where $\omega$ is the frequency of the laser light and $\omega_{p}$ is the plasma electron frequency. In this case, the self-focusing mechanism works due to the relativistic mass increase of the plasma electrons and the ponderomotive expulsion of electrons from the pulse region. Both effects lead to a local decrease in plasma frequency and an increase in the refractive index, which causes the medium to act as a positive lens [10].

For the fast ignition of ICF targets, one needs peta watt powers of laser beams. This is some order of magnitude

\footnotetext{
*Corresponding author. Also at the Department of Electromagnetic Energy Engineering, Osaka University, Yamada Oka 2-1, Suita, Osaka 565, Japan. Email address: katanaka@ile.osaka-u.ac.jp

${ }^{\dagger}$ Present address: Department of Nuclear Engineering, University of California at Berkeley, Etcheverry Hall, Berkeley, CA 947201730.

*Present address: Max-Planck-Institut für Quantenoptik HansKopfermann-Strasse 1, 85748 Garching, Germany.

${ }^{\S}$ Present address: Kansai Establishment, JAERI, Kizu, Kyoto 6190215, Japan.
}

higher than the critical power given by Eq. (1). Consequently, the laser beam could easily break in many filaments, each carrying the critical power [11], when propagating over hundreds of microns of the underdense corona surrounding the compressed core of the target. The initial seed for filamentation may have a different origin. The filamentation instability may start from nonuniformities in the laser transverse profile $[6,11]$. It may also be initiated by Weibel instability of background electrons pushed forward by the relativistically intense laser pulse $[10,12]$. The twodimensional (2D) PIC simulations of laser pulse filamentation just in front of the critical surface for plasma with a relatively sharp density gradient have been already reported [13]. Filamentation of longer laser pulses, caused by the ponderomotive mechanism, has been observed experimentally [14]. The filamentation may be undesirable, as it degrades the long single channel formation.

To date, most methods of observing channel formation experimentally have used probe beam interferometry $[14,15]$. This paper introduces a method of detecting the back-reflected light from an ultraintense laser as a possible means for diagnosing channel formation. The back-reflected image contains a number of bright spots which, we believe, are images of the laser filaments. This hypothesis is corrobo-

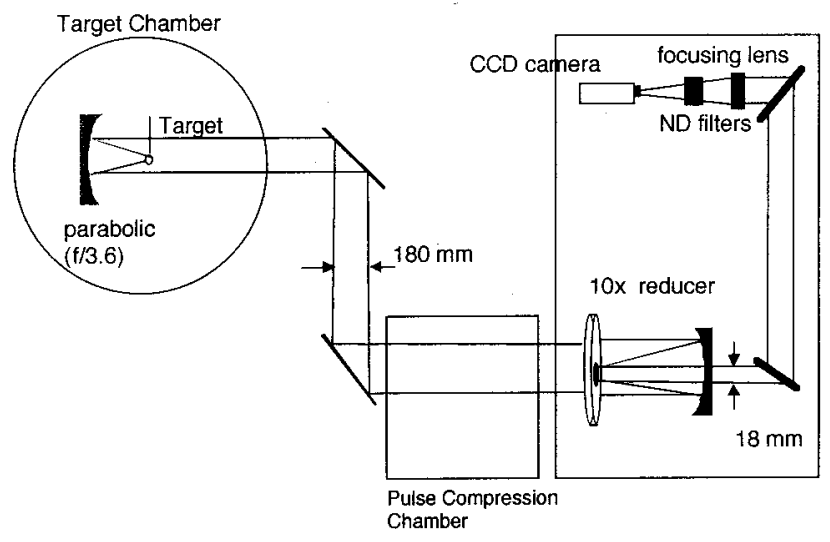

FIG. 1. Experimental setup at the back-reflected laser-light imaging system contains the parabolic mirror $(f / 3.6)$, the $10 \times$ reducer (two spherical mirrors), the focusing lens, nuetral density filters $(\mathrm{ND} 1 \times 3$, ND25, ND50), and the detector. 

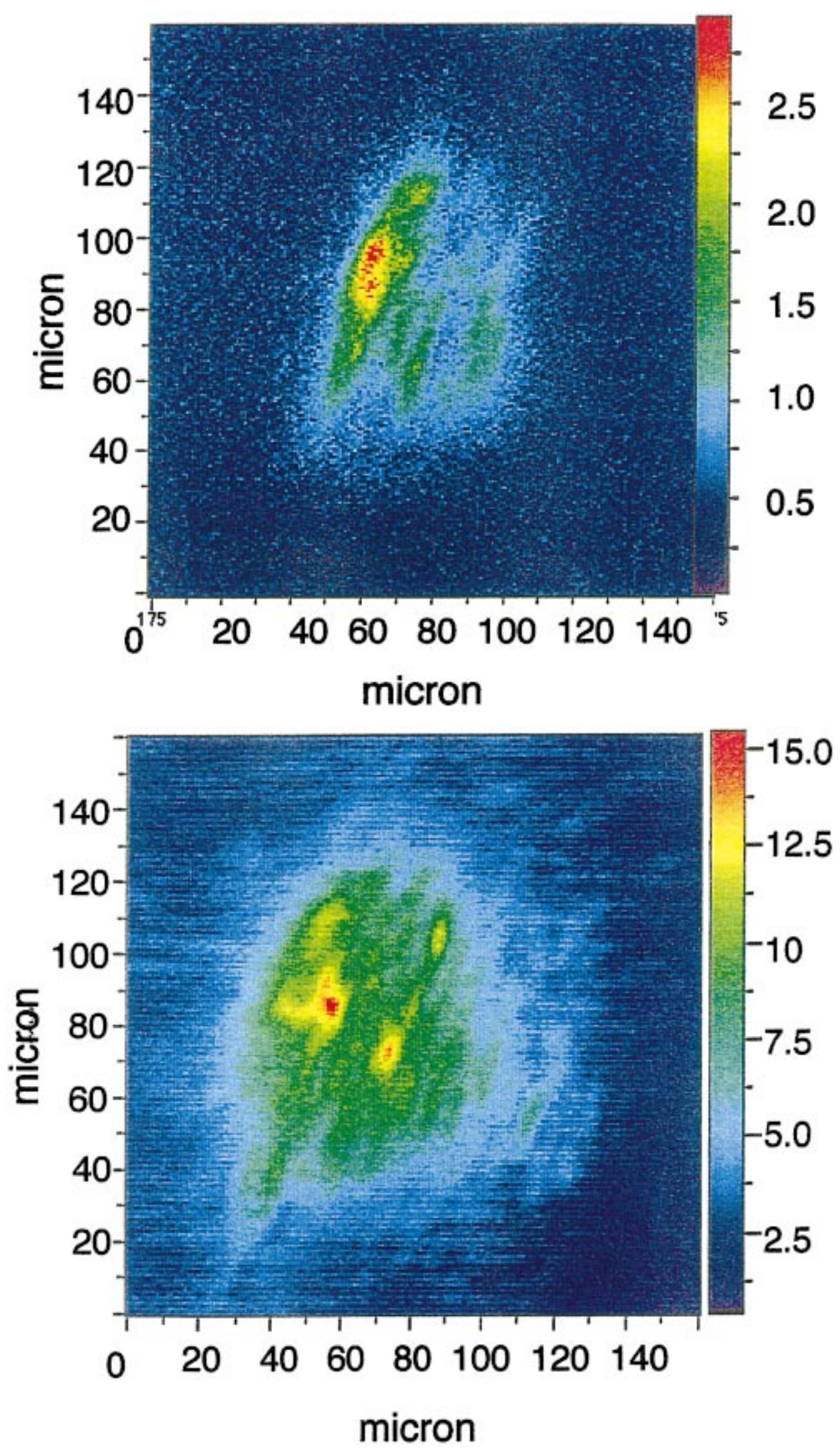

FIG. 2. (Color) (Top) Farfield pattern (FFP) of laser light reflecting off a 2.5-mm-diam metal sphere at surface focus. (Bottom) High-power shot on a planar metal target (molybdenum). Laser irradiated with $40.8 \mathrm{~J}$ energy, target at the best focus position.

rated by the full-scale three-dimensional particle-in-cell (PIC) simulations with the code VLPL (virtual laser plasma laboratory) [16]. The number of the observed filaments, however, is not given by the power ratio of $P / P_{c}$, but is limited by the plasma skin depth.

The experiments were carried out with the $\lambda$ $=1.053 \mu \mathrm{m}, 100 \mathrm{TW}$ laser system [17] at the Institute of Laser Engineering, Osaka University, Japan. The system is 
capable of delivering $50 \mathrm{~J}$ pulses on target with 0.5 ps pulse length. This laser system is synchronized with the GEKKO XII laser system to within a time jitter of less than 100 ps. Thus any of the laser system's 12 beams could be used to create preplasma at $\lambda=1.053 \mu \mathrm{m}$, with a pulse width from 0.1 to $1 \mathrm{~ns}$. The spot diameter of the short pulse was measured to be less than $30 \mu \mathrm{m}$ in vacuum giving a peak intensity on target of $10^{19} \mathrm{~W} / \mathrm{cm}^{2}$.

Shown in Fig. 1 is the experimental setup used to observe the back-reflected images presented in this paper. The laser light travels out of the pulse compression chamber towards the target vacuum chamber where it is focused by an $f / 3.6$ on-axis parabolic mirror. The mirror has a surface accuracy of $\lambda / 10$ for $1 \mu \mathrm{m}$ laser light. The mirror has a clear aperture of $190 \mathrm{~mm}$ and a focal length of $653 \mathrm{~mm}$. Due to the mirror's parabolic shape the curvature is not constant at the center the radius of curvature $r=1.3 \mathrm{~m}$. The mirror focusing ability is limited to approximately $10 \mu \mathrm{m}$ which makes the best focus spot size diameter three times the diffraction limit. The reflected light travels back down the beam line and into the detection system where it encounters a $10 \times$ reducer and is focused onto a charge-coupled device (CCD) camera which has a $480 \times 512$ pixel array at $11 \mu \mathrm{m}$ per pixel. Taking the ratio of the $f$ numbers from the cone of light onto the target and the cone of light onto the detector gives a magnification of 15.4 at the detector. The system has a resolution of better than $5 \mu \mathrm{m}$ with respect to the target. All images were analyzed in such a way that allowed us to take accurate measurements of the local photon count within specified areas of the captured image.

The far-field pattern (FFP) of a continuous-wave (cw) yttrium aluminum garnet (YAG) laser through the short pulse beam line was characterized by measuring the back-reflected light from a steel ball at surface focus. The image of this FFP is shown in Fig. 2 (top). The modulation seen in the image is perpendicular to the direction of polarization and is caused by imperfections in the holographic gratings setting for chirped pulse amplification inside the compression chamber. The image is approximately $45 \mu \mathrm{m}$ along the horizontal, and $20 \%$ of the intensity is contained in the bright spot on the left. Figure 2 (bottom) shows the backscattered image of a high power shot (energy equals $40.8 \mathrm{~J}$ ) focused onto the surface of a flat metal target. As seen from the images, the same modulation characteristics are present in both the high power shot and the cw YAG laser FFP. There is, however, the indication of a few new intense spots in Fig. 2 (bottom) compared to Fig. 2 (top). We consider these spots as a hint of self-focusing in a weak plasma caused by a prepulse. This type of CPA system has been known to have a very small amount of prepulses compared to the main pulse of the order of $10^{-3}$ to $10^{-4}$.

The parabolic mirror can be moved by means of stepper motors in order to focus the laser light behind or in front of the target plane, which is often necessary for laser-plasma experiments. The stepper motors have an error in the absolute amount of displacement of each axis $(X, Y$, and $Z)$ of less than $0.1 \%$. Their position reproducibility is better than a few microns. If the mirror is defocused to a position in front of the target surface, the resultant backscattered image cannot be called a FFP. The depth of focus of the parabolic mirror has been calculated to be $81 \mu \mathrm{m}$. Therefore, any image out-

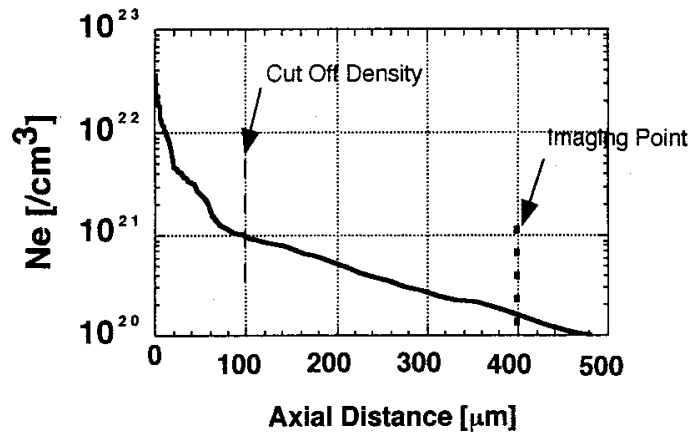

FIG. 3. Axial electron density profile of preplasma. The original target surface is at $z=0 \mu \mathrm{m}$. The dashed line indicates critical surface for $\lambda=1.053 \mu \mathrm{m}$ laser light. The dotted line indicates the plane of observation of the detection system.

side that range of defocus can only be referred to as the spatially resolved intensity distribution at the focal plane of the parabolic mirror.

To study the interaction effects on the backscattered image of the short pulse laser with a long scale length plasma, a preplasma was created on a solid target (either $\mathrm{CH}$ or $\mathrm{CD}$ ) by three of the GEKKO XII beams. The beams each consisted of a single pulse with a pulse width of $100 \mathrm{ps}, 0.53 \mu \mathrm{m}$ light in an overlapping region of $240 \mu \mathrm{m}$ diameter at an intensity of $2 \times 10^{15} \mathrm{~W} / \mathrm{cm}^{2}$. The beams heat the target in 1 $\mathrm{ns}$ before the high-intensity short pulse arrives.

The density profile along the laser axis was calculated in one dimension using the code ILESTA $[18,19]$ and is shown in Fig. 3. The density is plotted $1 \mathrm{~ns}$ after the preplasma pulse, just before the arrival of the short pulse. The original target surface is at $z=0 \mu \mathrm{m}$. The dashed line indicates the position of the critical surface for $1.053 \mu \mathrm{m}$ laser light at approximately $250 \mu \mathrm{m}$ from the surface of the target. The dotted line shows the focal plane (plane of observation) of the detection system due to the defocused position of the parabolic mirror.

The back-reflected image of a high power shot (energy is $50 \mathrm{~J}, 0.5 \mathrm{ps}$ ) focused onto a preplasma is shown in Fig. 4. The focal plane of the parabolic mirror was located at 400 $\mu \mathrm{m}$ in front of the target. The critical surface in the case is in $150 \mu \mathrm{m}$ behind the focal plane. The large-scale pattern of the image closely resembles the cw FFP. There is a distinctive difference between Fig. 4 with preformed plasma and Fig. 2(b) without preformed plasma. We clearly observe a number of small-scale high intensity spots as red areas in the image. These spots are $35 \%$ to $50 \%$ more intense than the surrounding green area. The seven most intense spots inside the original focal spot were measured to contain approximately 3\% each of the total back-reflected intensity. The hot spots appearance in Fig. 4 is a result of the ultraintense laserplasma interaction. The possible nonlinear processes are stimulated Raman scattering (SRS), stimulated Brillouin scattering (SBS), filamentation, and whole beam selffocusing. SRS and SBS are the interaction between incident laser electromagnetic and plasma or ion-acoustic waves. SRS causes nonlinear scattering around the fundamental frequency $\omega_{0}$ in the range $\pm 0.5 \omega_{0}$. The image was taken using a filter at the fundamental wavelength. The SRS close to the fundamental would correspond to scattering in very under- 


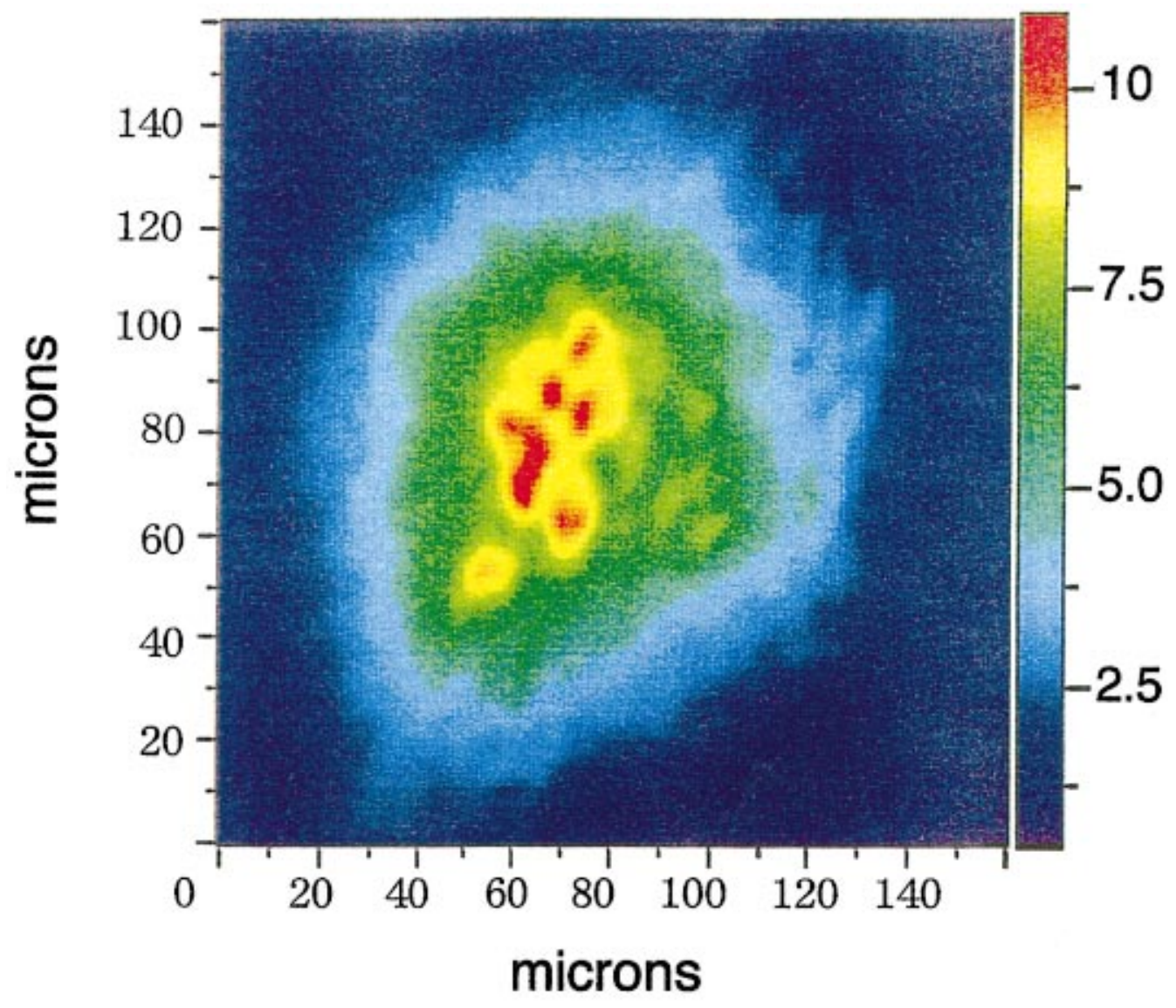

FIG. 4. (Color) Back-reflected image of high-power shot No. 21251 (energy is $50 \mathrm{~J}$, pulse width is $0.5 \mathrm{ps)}$. The presence of multiple, high intensity spots inside the original focal spot indicates filamentation.

dense plasma, far outside of the focal plane. Thus, SRS could not be observed as the small-scale spots. On the contrary, the downshift of SBS is small and redshifted by the ion-acoustic frequency. SBS may reflect the incoming laser light at a wavelength close to the fundamental. Although we may not exclude that both filamentation and whole beam selffocusing may drive SBS and the image in Fig. 4 might include a contribution from SBS, it is unlikely for SBS to create a transverse spatial modulation directly as shown in Fig. 4. It is more natural to suppose that the multiple hot spots observed in Fig. 4 are created, when the incoming laser pulse broke up into several filaments at a relativistic intensity. We believe that these bright spots are the evidence of the laser beam filamentation. The small transverse radius of the spots, $<5 \mu \mathrm{m}$, is close to the optical resolution limit. This indicates that the filaments exist from around the image plane (the vacuum focus position) to the reflection point (critical density or the head of the filaments). This kind of long filamentation structures is supported with the threedimensional (3D) PIC simulation as shown later. Adding the back-reflected intensity in all the hot spots, we estimate that about $20 \%$ of the laser power went into the production of filaments.

To investigate the possibility of laser beam filamentation, we have performed 3D particle-in-cell simulations using the code VLPL (Virtual Laser Plasma Laboratory) [15]. The simulations have been done for the experimental plasma density profile. The laser pulse was of $100 \mathrm{TW}$ power with a Gaussian temporal and spatial profile at the vacuum focal spot. The most intense part of the vacuum focal spot in the experiment was elliptical with approximately $20 \times 30 \mu \mathrm{m}$ as shown in Fig. 2, and we chose $\sigma_{x}=20 \mu \mathrm{m}$ and $\sigma_{y}$ $=30 \mu \mathrm{m}$. The on-axis intensity was $I_{0}=10^{19} \mathrm{~W} / \mathrm{cm}^{2}$. The laser beam was linearly polarized in the $Y$ direction. The ions have the deuteron charge-to-mass ratio. We initialized the laser pulse at $z=1000 \mu \mathrm{m}$ in front of the critical surface. The mesh cell size is $h_{x} \times h_{y} \times h_{z}$ with the transverse dimensions $h_{x}=h_{y}=0.4 \mu \mathrm{m}$ and the longitudinal one $h_{z}=0.1 \mu \mathrm{m}$. The transverse resolution is fine enough and the width of filaments is about ten times that of the pixel size. Thus, we exclude numerics as a reason or limit for the filamentation process. Later on we followed the laser pulse using the "moving window" [20-22] shifting the simulation domain together with the laser pulse. The "moving window" technique allows us to simulate the laser beam propagating over long distances while still fitting the simulation into the finite computer memory. The demand for the computer memory is proportional to the "window" size. The window must match the laser pulse length. Thus, the simulation of longer pulses is much more expensive than that of the shorter ones. This forced us to shorten the simulated pulse duration to $330 \mathrm{fs}$. It is $30 \%$ smaller than the actual laser pulse duration. This results in correspondingly lower energy of the laser beam in the simulation, as we maintain the power equal to the experimental one. Our aim, however, is not to reproduce the experiment down to the very detail, but to understand the physics of the observed filamentation. Although we start with a 

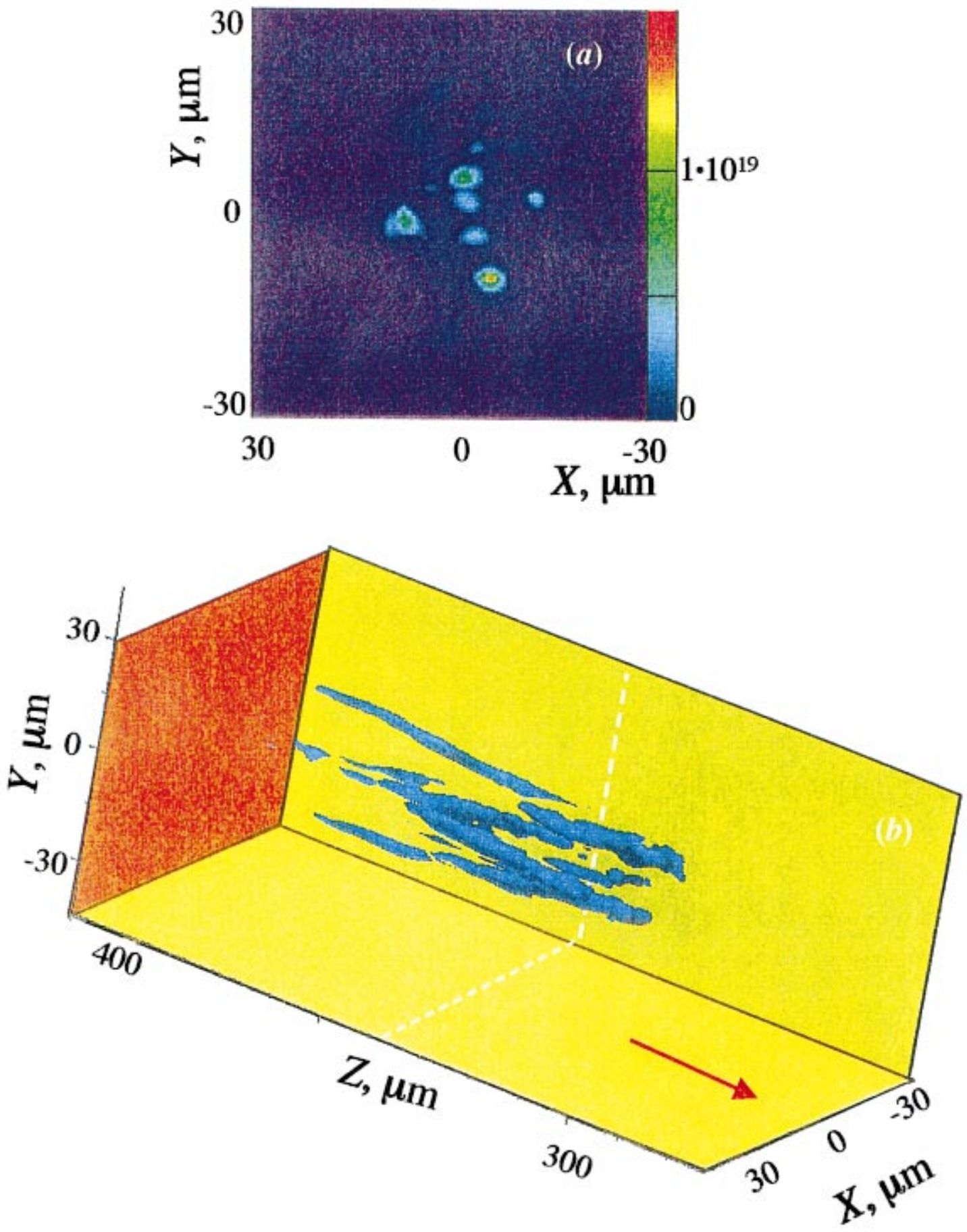

FIG. 5. (Color) 3D PIC simulation result for parameters close to the experimental ones. (a) Transverse $(Y$ - $Z)$ cross section of the laser beam intensity at the $z=250 \mu \mathrm{m}$ position $m$ in front of the critical surface and (b) a perspective view at the laser intensity isosurface of $\mathbf{I}$ $=5 \times 10^{18} \mathrm{~W} / \mathrm{cm}^{2}$ shows several well-developed filaments.

smooth transverse profile of the laser beam, we do observe filamentation. The filamentation process starts about $700 \mu \mathrm{m}$ in front of the critical surface, where the plasma density is $0.1 n_{c}$. After a stage of linear growth and later nonlinear interaction between the filaments [10], we observe several already well developed channels at $z=500 \mu \mathrm{m}$ in front of the critical surface. Figure 5(a) shows the transverse cut of the laser intensity at this position, and Fig. 5(b) provides the perspective view at the filamentation. The maximum intensity reaches $6 \times 10^{19} \mathrm{~W} / \mathrm{cm}^{2}$ in the strongest one. These filaments are already separated by several plasma skin depths $c / \omega_{p}$. This leads to an effective plasma screening, and the filaments propagate further independently from each other.

The threshold power needed for self-focusing at the plasma density $n=0.1 n_{c}$ is about $P_{\text {th }}=170 \mathrm{GW}$, as follows from Eq. (1). The actual laser power, $100 \mathrm{TW}$ exceeds it by factor 600 . This means that, in principle, a wide laser beam of this power could be split into 600 filaments. We, however, observe only a few of them both in experiment and in PIC simulations, though the spatial resolution of the diagnostics may not be good enough to observe very fine filaments with a $5 \mu \mathrm{m}$ spatial resolution. 
The small number of filaments can be understood in the following way. A hollow channel in plasma of density $n_{e}$ is able to confine without leakage an electromagnetic wave with the transverse wave number $k_{\perp}$. Waves with $k_{\perp}>k_{p}$ cannot be trapped in the the channel and spread into the surrounding plasma. This limits the minimum radius of the laser filament to the plasma skin depth, $\eta=R \omega_{p} / c$. If the channel is not evacuated completely, but contains some residual plasma, the maximum transverse number of the confined wave is even smaller, and the minimum radius of the filament is larger. Thus, even if the laser beam is linearly or nonlinearly focused down to a spot smaller than $c / \omega_{p}$ it could not be trapped and will diffract again. It is known also that when the distance between the filaments is comparable with the plasma skin depth, they may attract each other and merge $[10,23]$. This confluence of filaments is due to the currents of fast electrons accelerated forward by the intense laser light. These forward electron currents generate strong, up to $100 \mathrm{MG}$ magnetic fields [24,25] and attract each other. The relativistic electrons modify the plasma refraction index, and the light may follow the electrons [10]. If, however, the distance between the filaments is many plasma skin depths, the quasistatic magnetic fields are screened exponentially, and the filaments propagate independently. Thus, the mean separation between the surviving channels must be many plasma skin depths. All these effects prohibit the laser beam filamentation into numerous channels, and we observe only a few filaments in experiment and simulations. We expect that the decisive dimensionless parameter influencing the number of the formed filaments is the ratio of the laser beam radius to the plasma skin depth: $\eta=R \omega_{p} / c$. If a laser pulse with power many times higher than the critical (1) propagates through plasma, and the parameter $\eta=R \omega_{p} / c$ becomes large somewhere during the full interaction, one may expect a strong filamentation. Otherwise, the filamentation is prohibited and the beam self-focuses as a whole.

As a conclusion, we have observed filamentation of a relativistically strong laser pulse both in experiment and in the full-scale 3D PIC simulations. A laser beam with power many times above the self-focusing threshold filaments when its radius is significantly larger than the local plasma skin depth: $c / \omega_{p}$. However, the number of filaments is given not by the ratio of the beam power to the critical one, but is limited by the plasma skin depth.

The work of M.A. was supported in part by the William J. Fulbright Foundation. We acknowledge all the technical support of the engineering staff at the Institute of Laser Engineering (ILE) for the laser system operation, target fabrication, and data acquisition. A.P. thanks ILE for the hospitality during his three-month stay at Osaka University. The 3D PIC simulations have been performed on a 256 processor partition of the CRAY-T3E computer at Rechenzentrum Garching.
[1] D. Strickland and G. Mourou, Opt. Commun. 56, 219 (1985).

[2] G. A. Mourou, C. P. J. Barty, and M. D. Perry, Phys. Today 264, 917 (1994).

[3] M. Tabak, J. Hammer, M. Glinsky, W. L. Kruer, S. C. Wilks, J. Woodworth, E. Campbell, and M. D. Perry, Phys. Plasmas 1, 1626 (1994).

[4] G. Schmidt and W. Horton, Plasma Phys. Controlled Fusion 9, 85 (1985).

[5] G. Z. Sun, E. Ott, Y. C. Lee, and P. Guzdar, Phys. Fluids 30, 526 (1987).

[6] A. B. Borisov, O. B. Shiryaev, A. McPherson, K. Boyer, and C. K. Rhodes, Plasma Phys. Controlled Fusion 37, 569 (1995).

[7] P. Monot, T. Auguste, P. Gibbon, F. Jakober, G. Mainfray, A. Dulieu, M. Louis-Jacquet, G. Malka, and J. L. Miquel, Phys. Rev. Lett. 74, 2953 (1995).

[8] R. Wagner, S.-Y. Chen, A. Maksimchuk, and D. Umstadter, Phys. Rev. Lett. 78, 3125 (1997).

[9] R. Fedoseevs, X. F. Wang, and G. D. Tsakiris, Phys. Rev. E 56, 4615 (1997)

[10] A. Pukhov and J. Meyer-ter-Vehn, Phys. Rev. Lett. 76, 3975 (1996).

[11] F. Vidal and T. W. Johnston, Phys. Rev. Lett. 77, 1282 (1996).

[12] W. B. Mori, C. Joshi, J. M. Dawson, D. W. Forslund, and J. M. Kindel, in Laser Interaction and Related Plasma Phenomena, edited by H. Hora and G. Miley (Plenum, New York, 1986), Vol. 7, p. 767.

[13] S. Wilks and W. L. Kruer, IEEE J. Quantum Electron. 33, 1954 (1997).
[14] P. E. Young, Phys. Fluids B 3, 2331 (1991); Phys. Plasmas 2, 2815 (1995).

[15] K. A. Tanaka, H. Hashimoto, R. Kodama, Y. Sentoku, and K. Takahashi, Phys. Rev. E 60, 3283 (1999).

[16] A. Pukhov and J. Meyer-ter-Vehn, Bull. Am. Phys. Soc. 41, 1502 (1996).

[17] Y. Kato, Y. Kitagawa, K. A. Tanaka, R. Kodama, H. Fujita, T. Kanabe, T. Jitsuno, H. Shiraga, H. Takabe, M. Murakami, H. Nishimura, and K. Mima, Plasma Phys. Controlled Fusion 39, A145 (1997)

[18] H. Takabe, Phys. Fluids 31, 2284 (1988).

[19] H. Takabe and T. Ishii, Jpn. J. Appl. Phys., Part 1 32, 5675 (1993).

[20] W. B. Mori, IEEE J. Quantum Electron. 33, 1942 (1997).

[21] A. Pukhov and J. Meyer-ter-Vehn, Phys. Plasmas 5, 1880 (1998).

[22] G. A. Askar'an, S. V. Bulanov, F. Pegoraro, and A. Pukhov, Zh. Eksp. Teor. Fiz. Pis'ma Red. 60, 240 (1994) [JETP Lett. 60, 251 (1994)].

[23] M. Borghesi, A. J. Mackinnon, R. Gaillard, O. Willi, A. Pukhov, and J. Meyer-ter-Vehn, Phys. Rev. Lett. 80, 5137 (1998).

[24] G. A. Askar'an, S. V. Bulanov, F. Pegoraro, and A. Pukhov, Zh. Eksp. Teor. Fiz. Pis'ma Red. 60, 240 (1994) [JETP Lett. 60, 251 (1994)].

[25] M. Borghesi, A. J. Mackinnon, R. Gaillard, O. Willi, A. Pukhov, and J. Meyer-ter-Vehn, Phys. Rev. Lett. 80, 5137 (1998). 